УДК 334.021, 338.364, 339.18

$10.17213 / 2075-2067-2020-6-193-201$

\title{
ПЕРСПЕКТИВНЫЕ НАПРАВЛЕНИЯ РАЗВИТИЯ КОМПЛАЕНС В БИЗНЕСЕ В УСЛОВИЯХ ЦИФРОВОЙ ЭКОНОМИКИ
}

\author{
(C) 2020 г. Н. В. Рагулина, А. И. Литвинова
}

\section{Российский университет дружбы народов, г. Москва, Россия}

Цель исследования. В иентре внимания статьи находится проблема неопределенности сущңности и характера влияния циифровой экономики на бизнес-комплаенс. Статья нацелена на решение этой проблемы посредством определения перспективных направлений развития комплаенс в бизнесе в условиях ичифровой экономики.

Методология исследования. Для решения поставленной проблемы автором проводится комплексное экономико-математическое моделирование влияния различных факторов государственного и корпоративного управления иифровой экономикой на выраженность результатов в области бизнес-комплаенс с применением метода корреляиионного анализа.

Результаты исследования. B cmaтье обобщен международный onыт за 2020 2. Результаты моделирования позволили выявить перспективные направления развития комплаенс в бизнесе в условиях ичифровой экономики, а также проранжировать по степени значимости факторы управления, сформировав научную основу для повымения его эффективности.

Перспективы исследования. Вклад проведенного исследования в развитие теории и практики бизнес-комплаенс состочт в уточнении особенностей развития изучаемой практики в условиях ичифровой экономики. Доказанный значимый вклад ичифровых технологий и факторов управления ииффровой экономикой в достижение результатов бизнескомплаенс позволяет целенаправленно управлять этими факторами и получать более выраженные результаты. Это будет системно способствовать преодолению теневой экономики и формированию сочииальной рыночной экономики.

Ключевые слова: комплаенс; корпоративная социальная ответственность; бизнес; циирровая экономика; цчифровые технологии.

\section{PROMISING DIRECTIONS FOR DEVELOPMENT OF COMPLIANCE IN BUSINESS IN DIGITAL ECONOMY}

\author{
(C) $2020 \quad$ Y. V. Ragulina, A. I. Litvinova
}

\section{Peoples' Friendship University of Russia, Moscow, Russia}

Purpose of the research. The article focuses on the problem of the uncertainty of the essence and nature of the impact of the digital economy on business compliance. The article is aimed at solving this problem by identifying promising directions for the development of compliance in business in the digital economy.

Research methodology. To solve the problem posed, the author carries out a comprehensive economic and mathematical modeling of the influence of various factors of state and corporate 
governance of the digital economy on the severity of results in the field of business compliance using the method of correlation analysis.

Research results. The article summarizes the international experience for 2020. The modeling results made it possible to identify promising directions for the development of compliance in business in the digital economy, as well as to rank management factors according to the degree of importance, forming a scientific basis for increasing its effectiveness.

Research prospects. The contribution of the study to the development of the theory and practice of business compliance consists in clarifying the features of the development of the studied practice in the digital economy. The proven significant contribution of digital technologies and factors of digital economy management to the achievement of business compliance results allows you to purposefully manage these factors and get more pronounced results. This will systematically contribute to overcoming the shadow economy and the formation of a social market economy.

Key words: compliance; corporate social responsibility; business; digital economy; digital technologies.

Введение. Прогрессивной формой проявления корпоративной социальной ответственности является комплаенс. Он находится на стыке и воплощает в себе и полномасштабное соблюдение действующего нормативно-правового обеспечения, и следование интересам акционеров в менеджменте бизнеса, и учет мнений заинтересованных лиц - paботников, потребителей и общества. Сочетание черт официального (не теневого) бизнеса и ответственного (инициативного) бизнеса обеспечивает широкие границы комплаенс и позволяет сделать его массовой практикой в современном бизнесе.

Хотя интерес к комплаенс большинство предприятий проявляет на протяжении многих десятилетий, его массовое внедрение в хозяйственную практику сдерживалось недостаточной информационной поддержкой, обуславливающей неясность в отношении требований и государства (из-за сложности уследить за изменяющимся законодательством), и акционеров (из-за недостаточного контакта с ними), и потребителей (из-за барьеров сбора обратной связи), комплаенс традиционно был доступен только для наиболее крупного бизнеса.

В условиях цифровой экономики созданы благоприятные возможности для развития комплаенс - цифровые технологии позволяют преодолеть информационные барьеры и предоставить все необходимые данные для полномасштабного комплаенс всех предпри- ятий. Однако, проблема заключается в неопределенности того, какие направления комплаенс могут получить наибольшее развитие с помощью цифровых технологий, а также какие меры государственного и корпоративного управления наиболее предпочтительны и должны быть предприняты.

Эта статья нацелена на решение поставленной проблемы и исследование перспективных направлений развития комплаенс в бизнесе в условиях цифровой экономики, а также определение рамочных требований к государственному и корпоративному управлению с применением цифровых технологий в интересах развития бизнес-комплаенс.

Материалы и методология. Различные вопросы комплаенс и перспективы его применения в современном бизнесе достаточно подробно освещены в трудах таких ученых, как Т.А. Абрамян [1], С.Н. Еремина [2], О.Г. Карпович, Ю.В. Трунцевский [3], В.Е. Понаморенко, Д.Г. Коровяковский, А.А. Тыртышный [4], Е.Г. Попкова [5], В.И. Прасолов, А.К. Чешунас [6], Ю.Н. Румянцева [7] и А.А. Сапфирова [8]. При этом вклад цифровых технологий в развитие комплаенс недостаточно подробно исследован и нуждается в дальнейшем углубленном изучении для заполнения этого пробела в научных знаниях.

Методология исследования базируется на применении экономико-математического 
аппарата, в частности, метода корреляционного анализа. Для получения наиболее достоверных результатов исследование вклада цифровых технологий в развитие комплаенс в бизнесе осуществляется на базе международной выборки, состав стран для которой определен по критерию уровня экономической свободы. Для обеспечения репрезентативности выборки в нее включено по 2 страны из каждого класса в соответствии с материалами [9]. Это позволяет исключить влияние государственного регулирования и механизма рынка на развитие комплаенс, тем самым получив наиболее достоверные результаты, очищенные от искажающего воздействия сторонних факторов. Экономическая свобода стран выборки проиллюстрирована на рис. 1.

Как наглядно продемонстрировано на рисунке 1, разрыв в уровне экономической свободы в странах выборки достаточно велик, чтобы действительно изолировать ее от влияния государственного регулирования и механизма рынка. Так, в Боливии соответствующий индекс составляет 42,8 баллов, а в Сингапуре 89,4 балла, то есть на 108,88\% (более, чем в 2 раза) больше.
На основе сформированной выборки стран в статье рассчитывается корреляционная зависимость между результатами в области комплаенс и отобранными потенциально влияющими на них факторами цифровой экономики.

Результаты в области комплаенс количественно измерены и выражены с помощью показателей из отчета [10], среди которых регулирование конфликта интересов (п. 1.18), сила стандартов аудита и бухгалтерского учета (п. 1.17), защита прав работников (п. 8.06) и многостороннее сотрудничество (п. 12.04). Факторы цифровой экономики, потенциально влияющие на результаты в области комплаенс, включают в себя такие показатели отчета [10], как адаптация правовой базы к цифровым бизнес-моделям (п. 1.22), электронное гражданское общество (п. 1.12) и цифровые навыки активного населения (п. 6.05), а также такой показатель из отчета [11], как цифровой бизнес (business agility). Эмпирические данные об уровне развития бизнес-комплаенс и влияющих на него факторах цифровой экономики в странах выборки в 2020 г. представлены в табл. 1.

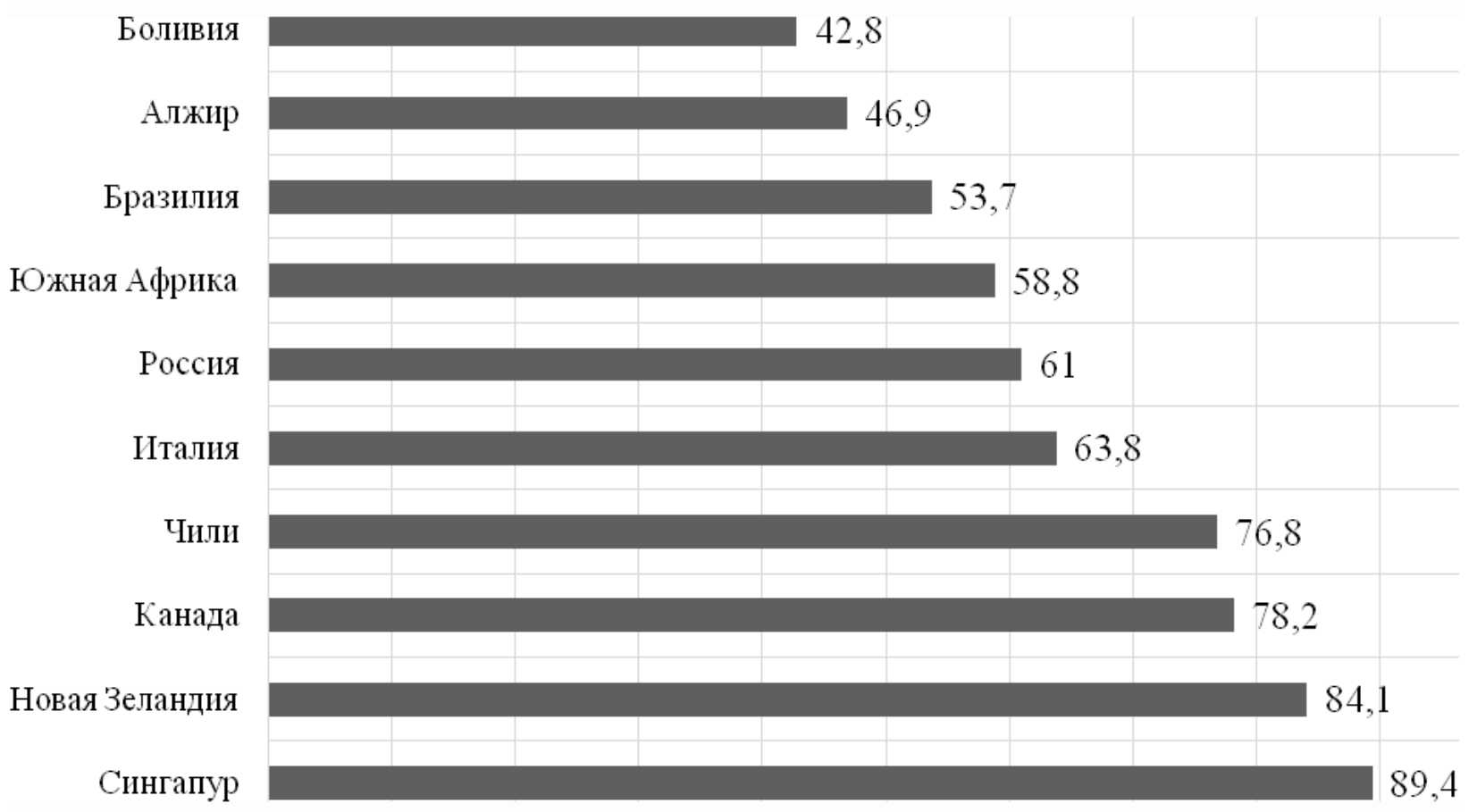

Рис. 1. Характеристика выборки стран по уровню экономической свободы в 2020 г., баллы 1-100 [9] 
Результаты и их обсуждение. Обработка данных из табл. 1 позволила получить следующие результаты, отражающие корреляционную связь рассматриваемых показателей и охарактеризовать влияние цифровой экономики на бизнес-комплаенс (рис. 2, 3, 4).

Принимая во внимание детализированные результаты проведенного корреляционного анализа, представленные на рис. 2, можно утверждать, что перспективы развития комплаенс в бизнесе в условиях цифровой экономики достаточно широки и охватывают все его основные направления, реализуемые сегодня. Вместе с этим выявлена важность всех факторов цифровой экономики, предположение о значимости каждого из которых подтвердилось.

Наиболее сильная корреляция выявлена в отношении влияния адаптации правовой базы к цифровым бизнес-моделям (фактор государственного регулирования цифрового бизнеса) на развитие многостороннего сотрудничества бизнеса как направление комплаенс (учет мнений заинтересованных сторон (корреляция 92,69\%)). Также большая корреляция выявлена в отношении влияния цифровых навыков экономически активного населения на многостороннее сотрудничество $(76,94 \%)$.

Следует отметить, что отрицательные значения коэффициентов корреляции результатов в области бизнес-комплаенс с цифровым бизнесом свидетельствуют о позитивном влиянии этого фактора, поскольку он измеряется в местах, и потому его значение улучшается по мере уменьшения в противоположность остальным показателям, что и определяет полученные

Эмпирические данные об уровне развития бизнес-комплаенс и влияющих на него факторах цифровой экономики в странах выборки в 2020 г. $[10,11]$

\begin{tabular}{|c|c|c|c|c|c|c|c|c|c|}
\hline \multirow[b]{2}{*}{ 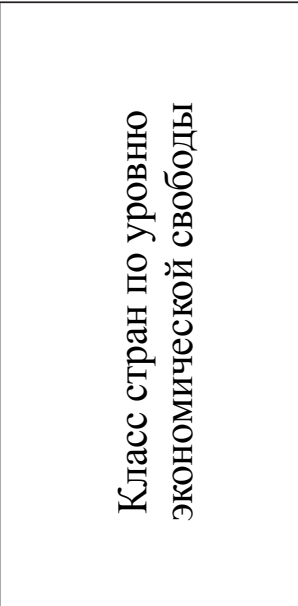 } & \multirow[b]{2}{*}{ Страна } & \multicolumn{4}{|c|}{$\begin{array}{c}\text { Результаты } \\
\text { в области комплаенс }\end{array}$} & \multicolumn{4}{|c|}{$\begin{array}{c}\text { Факторы цифровой экономики, } \\
\text { влияющие на результаты }\end{array}$} \\
\hline & & 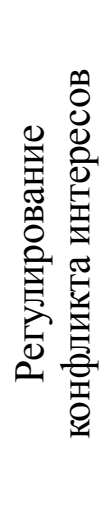 & 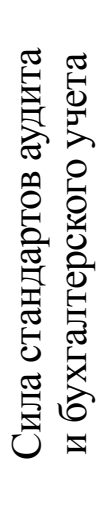 & 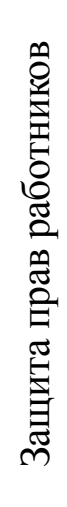 & 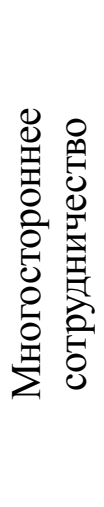 & 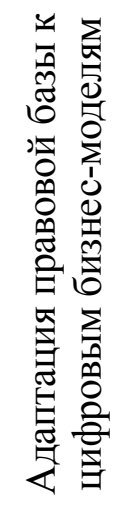 & 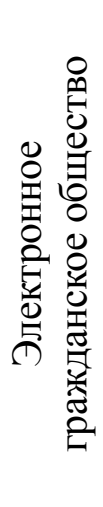 & 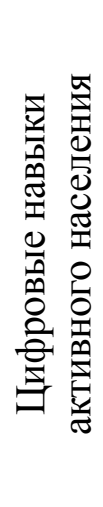 & 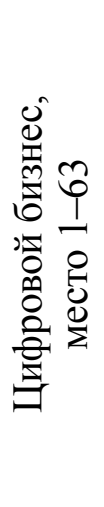 \\
\hline \multirow[b]{2}{*}{ Абсолютная } & Сингапур & 93 & 87,6 & 89 & 66 & 76,5 & 96,6 & 76,4 & 11 \\
\hline & $\begin{array}{l}\text { Новая } \\
\text { Зеландия }\end{array}$ & 93 & 84,7 & 89 & 61,4 & 65,1 & 98,3 & 65,5 & 46 \\
\hline \multirow{2}{*}{ Преобладающая } & Канада & 87 & 81,6 & 79 & 63,8 & 58 & 91 & 67,9 & 16 \\
\hline & Чили & 70 & 77,4 & 70 & 44,8 & 51,4 & 54,4 & 54,4 & 54 \\
\hline \multirow{2}{*}{ Ограниченная } & Италия & 57 & 57,2 & 98 & 45,6 & 36,1 & 95,5 & 52,9 & 23 \\
\hline & Россия & 50 & 54,6 & 77 & 49,5 & 48,1 & 92,1 & 65,8 & 60 \\
\hline \multirow[t]{2}{*}{ Минимальная } & $\begin{array}{l}\text { Южная } \\
\text { Африка }\end{array}$ & 80 & 67,5 & 86 & 52,6 & 42,4 & 84,8 & 37,9 & 58 \\
\hline & Бразилия & 57 & 61,1 & 62 & 44,3 & 33,5 & 97,2 & 34,8 & 41 \\
\hline \multirow{2}{*}{ Отсутствующая } & Алжир & 33 & 47,4 & 57 & 42,7 & 33,9 & 20,2 & 49,5 & - \\
\hline & Боливия & 70 & 61,1 & 72 & 31,1 & 16,3 & 57,9 & 37 & - \\
\hline
\end{tabular}


отрицательные значения. Обобщенная корреляция получена с помощью расчета средних арифметических, а показатели связи с цифровым бизнесом взяты с обратным знаком (рис. 3,4$)$.

Исходя из рис. 3 , можно сказать, что многостороннее сотрудничество представляет собой наиболее перспективное направление развития комплаенс в бизнесе в условиях цифровой экономики - среднее арифметическое коэффициентов корреляции составило $69,99 \%$. На втором месте по перспективам развития на базе цифровых технологий оказалось такое направление бизнес-комплаенс, как усиление стандартов аудита и бухгалтерского (финансового, корпоративного) учета и отчетности $(55,42 \%)$. Менее выраженными, но также перспективными оказались направления, связанные с совершенствованием практики регулирования конфликта интересов $(49,60 \%)$ и с усилением защиты прав работников $(48,05 \%)$.

Несмотря на выявленные некоторые различия во влиянии цифровых технологий, можно с уверенностью утверждать, что все рассмотренные направления развития бизнес-комплаенс могут получить импульс к развитию на базе новых возможностей, открывающихся в условиях цифровизации хозяйственных систем.
Как показали обобщенные результаты корреляционного анализа на рис. 4, наибольший вклад в развитие бизнес-комплаенс в современной мировой экономике вносит такой фактор цифровизации, как адаптация правовой базы к цифровым бизнес-моделям (70,32\%). Также достаточно значимыми оказались такие факторы, как электронное гражданское общество $(56,03 \%)$ и цифровые навыки активного населения $(54,13 \%)$. Наименее значимым, но также важным фактором, который должен приниматься во внимание, стал цифровой бизнес $(42,58 \%)$.

Это указывает, с одной стороны, на возможность диверсифицировать практики государственного и корпоративного управления развитием бизнес-комплаенс на базе возможностей цифровой экономики, с другой стороны, на доступность приоритезации доступных практик в интересах повышения эффективности управления ими при распределении инициатив и ресурсов.

Заключение. Завершая проведенное исследование, можно сделать вывод о том, что в условиях цифровой экономики открываются новые возможности для развития бизнес-комплаенс, действительно позволяющие сделать его массово доступным. Перспектив-

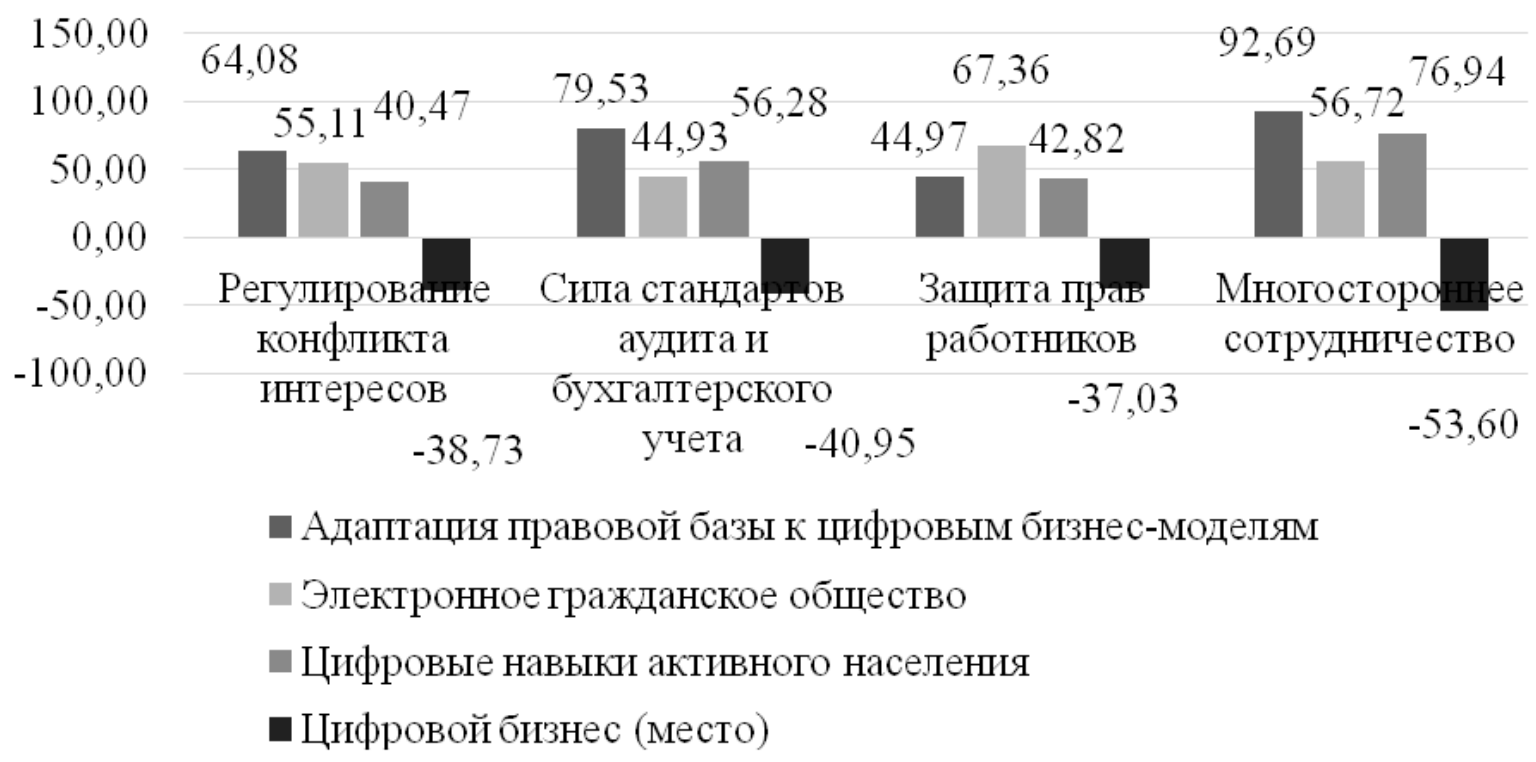

Рис. 2. Детализированная корреляция развития бизнес-комплаенс и влияющих на него факторов цифровой экономики в странах выборки в 2020 г. 


\section{Регулирование \\ конфликта \\ интересов}

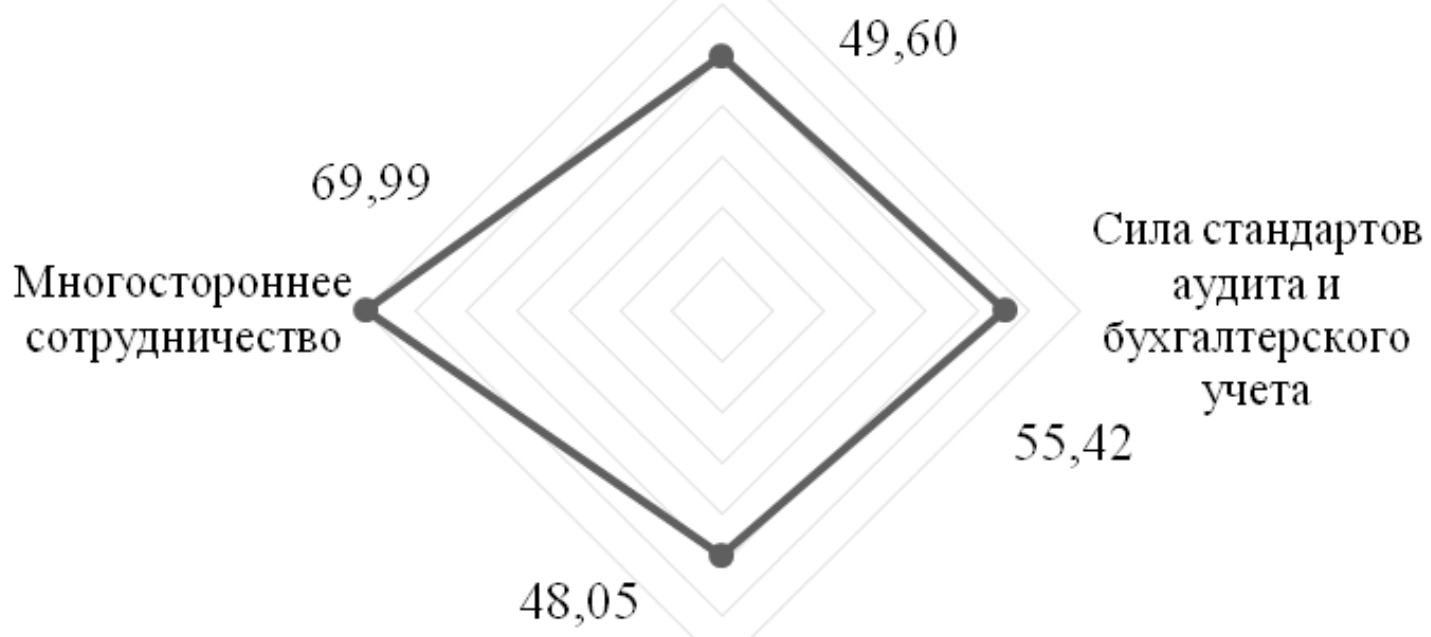

\section{Защита прав \\ работников}

Рис. 3. Обобщенная корреляция развития бизнес-комплаенс

под системным влиянием цифровой экономики в странах выборки в 2020 г.

\section{Адаптация правовой базы}

к цифровым бизнес-моделям

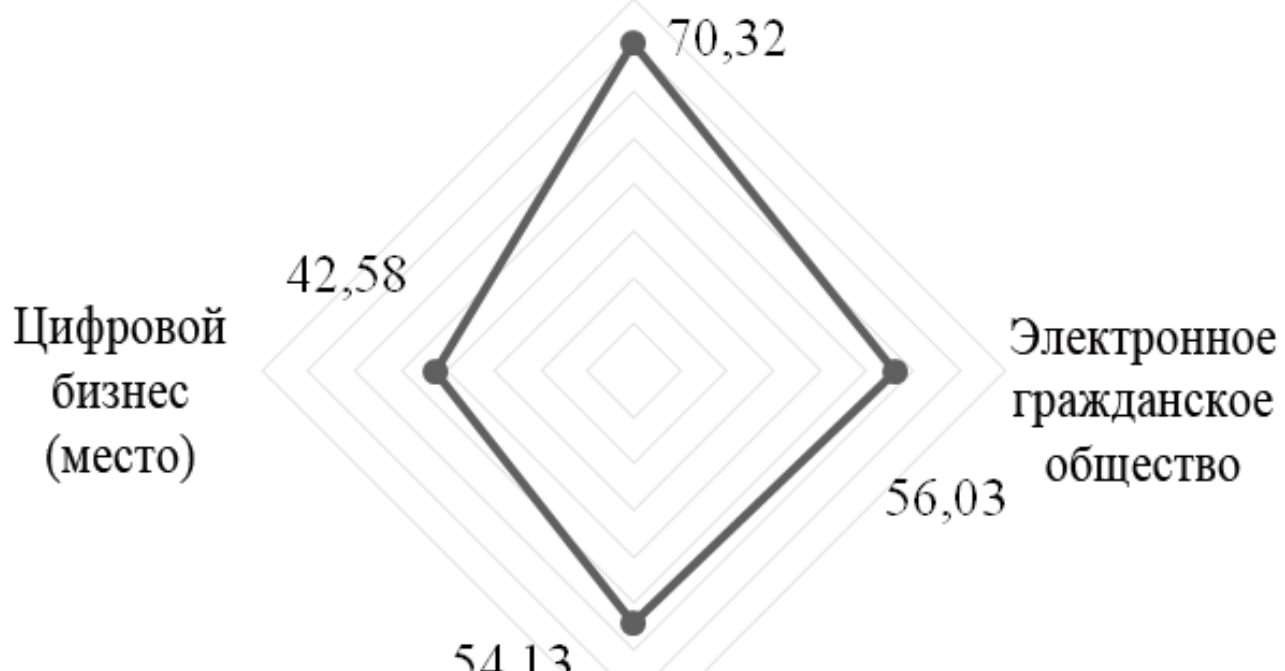

54,13

\section{Цифровые навыки \\ активного населения}

Рис. 4. Обобщенная корреляция влияния каждого фактора цифровой экономики на системное развитие бизнес-комплаенс в странах выборки в 2020 г. 
ные направления развития комплаенс в бизнесе в условиях цифровой экономики не ограничены и охватывают все существующие практики комплаенс, реализуемые в бизнесе. Тем не менее, данные перспективы наиболее выражены в отношении расширения многостороннего сотрудничества, корреляция которого с совокупностью факторов цифровой экономики оказалась наиболее высока и составила 69,99\%.

При управлении цифровой экономикой в интересах развития бизнес-комплаенс целесообразно уделять основное внимание государственному (внешнему) управлению, которое рекомендуется направить на освоение цифровых навыков широкими массами населения, становлением цифрового гражданского общества и модернизацией нормативно-правового обеспечения к потребностям и особенностям цифрового бизнеса. При этом возможности цифрового бизнеса ограничены и наименее выражены среди обозначенных факторов цифровой экономики.

Вклад проведенного исследования в развитие теории и практики бизнес-комплаенс состоит в уточнении особенностей развития изучаемой практики в условиях цифровой экономики. Доказанный значимый вклад цифровых технологий и факторов управления цифровой экономикой в достижение результатов бизнес-комплаенс позволяет целенаправленно управлять этими факторами и получать более выраженные результаты. Это будет системно способствовать преодолению теневой экономики и формированию социальной рыночной экономики.

\section{Литература}

1. Абрамян T.A. К вопросу о необходимости использования системы «комплаенс» в российском бизнесе / Т. А. Абрамян / Юрист. - 2017. — №17. - С. 19-21.

2. Еремина С.Н. Комплаенс в России: сущность и применение в сфере регулирования труда / С.Н. Еремина // Юрист. 2017. — №17. - С. 37-40.

3. Карпович О.Г. Особенности методологии современных комплаенс-программ / О.Г. Карпович, Ю.В. Трунцевский // Юрист. - 2017. — №18. - С. 9-15.
4. Понаморенко В.E. Комплаенс как фактор эффективности правового регулирования во внешней торговле / В.Е. Понаморенко, Д.Г. Коровяковский, А. А. Тыртышный // Таможенное дело. — 2017. — №3. C. $15-17$.

5. Попкова Е.Г. Роль индустрии 4.0 в обеспечении глобальной конкурентоспособности экономики современной России / Е. Г. Попкова // Экономика. Бизнес. Банки. 2018. - №5 (26). - С. 118-131.

6. Прасолов В. И. Аутсорсинг комплаенспроцедур как способ оптимизации расходов на комплаенс-функцию / В.И. Прасолов, A. К. Чешунас // Russian Economic Bulletin. 2020. - T. 3. - №2. - C. 19-24.

7. Румянцева Ю.Н. Антимонопольный комплаенс как часть комплаенс-программы соблюдения законодательства Российской Федерации / Ю.Н. Румянцева // Пролог: журнал о праве. - 2019. - №2 (22). - С. 53-56.

8. Сапфирова A.A. Трудовой комплаенс как способ предупреждения нарушений трудовых прав / А.А. Сапфирова // Теория и практика общественного развития. 2017. — №9. - C. 28-31.

9. Index of Economic Freedom 2020 [Электронный pecypc] // The Heritage Foundation. - Режим доступа: https://www. heritage.org/index/about (Дата обращения: 19.11.2020).

10. The Global Competitiveness Report 2019 [Электронный ресурс] // World Economic Forum. - Режим доступа: https://www. weforum.org/reports/how-to-end-a-decade-oflost-productivity-growth (Дата обращения: 19.11.2020).

11. World Digital Competitiveness Ranking 2019 [Электронный ресурс] // IMD. - Peжим доступа: https://www.imd.org/wcc/worldcompetitiveness-center-rankings/world-digitalcompetitiveness-rankings-2019/ (Дата обращения: 19.11.2020).

\section{References}

1. Abramjan T.A. K voprosu o neobhodimosti ispol'zovanija sistemy «komplaens» V rossijskom biznese [To the question on necessity of use of the system "compliance» in Russian business] / T. A. Abramjan / Jurist [The Lawyer]. 2017. — №17. — Pp. 19-21. 
2. Eremina S.N. Komplaens v Rossii: sushhnost' i primenenie $\mathrm{v}$ sfere regulirovanija truda [Compliance in Russia: the essence and application in the field of regulation of labor] / S. N. Eremina // Jurist [The Lawyer]. — 2017. №17. - Pp. 37-40.

3. Karpovich O. G. Osobennosti metodologii sovremennyh komplaens-programm [Peculiarities of the methodology of modern compliance programmes] / O.G. Karpovich, Ju. V. Truncevskij // Jurist [The Lawyer]. — 2017. №18. - Pp. 9-15.

4. Ponamorenko V.E. Komplaens kak faktor jeffektivnosti pravovogo regulirovanija vo vneshnej torgovle [Compliance as a factor in the effectiveness of legal regulation in foreign trade] / V.E. Ponamorenko, D. G. Korovjakovskij, A.A. Tyrtyshnyj // Tamozhennoe delo [Customs]. - 2017. — №3. - Pp. 15-17.

5. Popkova E. G. Rol' industrii 4.0 v obespechenii global'noj konkurentosposobnosti jekonomiki sovremennoj Rossii [The Role of industry 4.0 in ensuring the global competitiveness of the economy of modern Russia] / E. G. Popkova // Jekonomika. Biznes. Banki [Economy. Business. Banks]. — 2018. — №5 (26). Pp. 118-131.

6. Prasolov V.I. Autsorsing komplaensprocedur kak sposob optimizacii rashodov na komplaens-funkciju [Outsourcing of compliance procedures as a way to optimize expenses for the compliance function] / V. I. Prasolov, A. K. Ches- hunas // Russian Economic Bulletin. - 2020. Vol. 3. — №2. - Pp. 19-24.

7. Rumjanceva Ju. N. Antimonopol'nyj komplaens kak chast' komplaens-programmy sobljudenija zakonodatel'stva Rossijskoj Federacii [Antimonopoly compliance as part of the compliance program for compliance with the legislation of the Russian Federation] / Ju. N. Rumjanceva // Prolog: zhurnal o prave [Prologue: journal of law]. — 2019. — №2 (22). Pp. 53-56.

8. Sapfirova A.A. Trudovoj komplaens kak sposob preduprezhdenija narushenij trudovyh prav [Labor compliance as a way to prevent violations of labor rights] / A.A. Sapfirova // Teorija i praktika obshhestvennogo razvitija [Theory and practice of social development]. — 2017. №9. - Pp. 28-31.

9. Index of Economic Freedom 2020 [Jelektronnyj resurs] // The Heritage Foundation. URL: https://www.heritage.org/index/about (Date accessed: 19.11.2020).

10. The Global Competitiveness Report 2019 [Jelektronnyj resurs] // World Economic Forum. - URL: https://www.weforum.org/reports/how-to-end-a-decade-of-lost-productivitygrowth (Date accessed: 19.11.2020).

11. World Digital Competitiveness Ranking 2019 [Jelektronnyj resurs] // IMD. — URL: https://www.imd.org/wcc/world-competitivenesscenter-rankings/world-digital-competitivenessrankings-2019/ (Date accessed: 19.11.2020). 


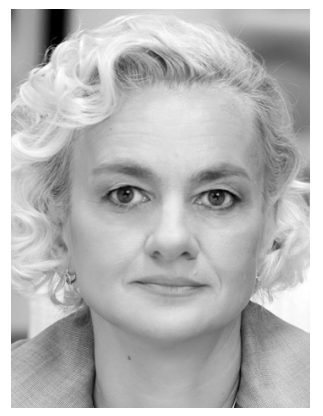

Рагулина Юлия Вячеславовна - доктор экономических наук, профессор, заведующая кафедрой комплаенса и контроллинга Российского университета дружбы народов. Почетный работник науки и техники Российской Федерации, Почетный работник высшего профессионального образования. Автор более 200 публикаций, ряда монографических исследований по государственному контролю и аудиту, государственному и муниципальному управлению, экономике муниципальных образований, внесла значительный научный вклад в обоснование основополагающих исследований по взаимодействию государства и бизнеса. Имеет монографии, подготовленные совместно с коллегами Гарварда.

Ragulina Yulia Vyacheslavovna - Doctor of Economic Sciences, Professor, Head of the Department of Compliance and Controlling at the Peoples' Friendship University of Russia. Honorary worker of science and technology of the Russian Federation, Honorary worker of higher professional education. Author of more than 200 publications, a number of monographic studies on state control and audit, state and municipal management, the economy of municipalities, made a significant scientific contribution to the foundation of fundamental research on the interaction of the state and business. He has monographs prepared jointly with Harvard colleagues.

117198, г. Москва, ул. Миклухо-Маклая, 6 6 Miklukho-Maklaya st., 117198, Moscow, Russia E-mail: julra@list.ru

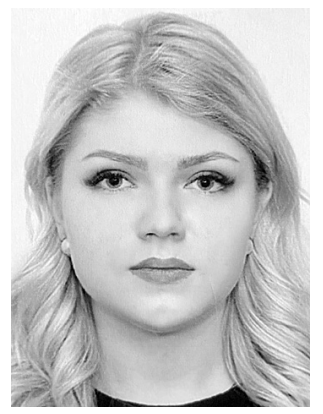

Литвинова Ангелина Ивановна - магистрант Российского университета дружбы народов.

Litvinova Angelina Ivanovna - Master's Student, Peoples' Friendship University of Russia.

117198, г. Москва, ул. Миклухо-Маклая, 6 6 Miklukho-Maklaya st., 117198, Moscow, Russia E-mail: ang1358@yandex.ru 